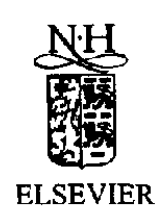

Nuclear Physics B (Proc. Suppl.) 104 (2002) 251-253

\title{
Topological Generalizations of Supersymmetry
}

\author{
K. Aghababaei Samani ${ }^{a}$ and A. Mostafazadeh ${ }^{b}$ \\ aInstitute for Advanced Study in Basic Sciences, 45195-159 Gava Zang, Zanjan, Iran \\ bDepartment of Mathematics, Koç University, Rumelifeneri Yolu, 80910 Sariyer, Istanbul, Turkey
}

We introduce a class of generalizations of supersymmetry that involve a set of integer-valued topological invariants. These symmetries are defined in terms of certain conditions on the spectral properties of the corresponding quantum systems. We identify the operator algebras of the generators of these symmetries and show that the algebras of ordinary and fractional supersymmetric quantum mechanics and parasupersymmetric quantum mechanics of order two are special cases of these operator algebras.

\section{Motivation}

Supersymmetric quantum mechanics has been originally developed by Witten as a toy model for understanding the phenomenon of supersymmetry breaking in supersymmetric field theories. This toy model has since become a source of new developments in mathematical physics. Among the most fascinating of these developments is the supersymmetric proof of the Atiyah-Singer index theorem. The main motivation for the present work is to extract the very basic features of supersymmetry that leads to its topological properties and to generalize supersymmetry to a larger class of symmetries with similar topological properties. We term these symmetries the Topological Quantum Symmetries or Topological Symmetries (TS).

\section{What Makes the Witten Index a Topo- logical Invariant?}

The Witten index can be defined for any $\mathscr{Z}_{2^{-}}$ graded Hilbert space $\mathcal{H}=\mathcal{H}_{+} \oplus \mathcal{H}_{-}$according to

indexwitten $=n_{+}^{(0)}-n_{-}^{(0)}$,

where $n_{ \pm}^{(0)}$ is the number of zero-energy states with grading \pm . The grading of the Hilbert space is achieved by a grading operator $\tau$ satisfying

$\tau^{\dagger}=\tau=\tau^{-1}$,

$\left.\mathcal{H}_{ \pm}=\left\{|\psi\rangle_{ \pm} \in \mathcal{H}|\tau| \psi\right\rangle_{ \pm}= \pm|\psi\rangle_{ \pm}\right\}$.
What makes the Witten index a topological invariant is the particular degeneracy structure of the supersymmetric systems, namely that the energy spectrum is nonnegative and the positive energy eigenstates come in pairs of opposite grading. These degeneracy properties follow from the algebraic structure of supersymmetry. The same is true for parasupersymmetry of order two [1]. In fact, as shown in Refs. [2,3], one can identify a class of parasupersymmetric systems that involve certain integer-valued topological invariants. In Refs. [4,5], we give a thorough treatment of the idetification and characterization of the general symmetries that lead to topological invariants similar to the Witten index. The present paper is a survey of our main results.

\section{What is a Topological Symmetry?}

Definition 1: Let $n$ be an integer greater than $1, \mathcal{H}$ denote the Hilbert space of a quantum system, and $\mathcal{H}_{1}, \mathcal{H}_{2}, \cdots, \mathcal{H}_{n}$ be (nontrivial) subspaces of $\mathcal{H}$. Then a state vector is said to have definite color $c_{\ell}$ iff it belongs to $\mathcal{H}_{\ell}$.

Definition 2: A quantum system is said to be $\mathbb{Z}_{n}$-graded iff its Hilbert space is the direct sum of $n$ of its (nontrivial) subspaces $\mathcal{H}_{\ell}$, and its Hamiltonian has a complete set of eigenvectors with definite color.

Definition 3: Let $m_{\ell}$ be positive integers for all $\ell \in\{1,2, \cdots, n\}$, and $m:=\sum_{\ell=1}^{n} m_{\ell}$. Then a quantum system is said to possess a $\mathbb{Z}_{n}$-graded topological symmetry of type $\left(m_{1}, m_{2}, \cdots, m_{n}\right)$ iff 
the following conditions are satisfied.

a) The quantum system is $\mathbb{Z}_{n}$-graded;

b) The energy spectrum is nonnegative;

c) For every positive energy eigenvalue $E$, there is a positive integer $\lambda_{E}$ such that $E$ is $\lambda_{E} m$-fold degenerate, and the corresponding eigenspaces are spanned by $\lambda_{E} m_{1}$ vectors of color $c_{1}, \lambda_{E} m_{2}$ vectors of color $c_{2}$, $\cdots$, and $\lambda_{E} m_{n}$ vectors of color $c_{n}$.

Theorem: Consider a quantum system possessing a $\mathbb{Z}_{n}$-graded topological symmetry of type $\left(m_{1}, m_{2}, \cdots, m_{n}\right)$, and let $n_{\ell}^{(0)}$ denote the number of zero-energy states of color $c_{\ell}$. Then for all $i, j \in\{1,2, \cdots, n\}$, the integers

$\Delta_{i j}:=m_{i} n_{j}^{(0)}-m_{j} n_{i}^{(0)}$

remain invariant under continuous symmetrypreserving changes of the quantum system.

Proof: See Ref. [5].

\section{Algebraic Structure of Topological Sym- metry}

Given the above definition of a topological symmetry, we have obtained the algebraic structures (operator algebras) supporting these symmetries. Our derivation of the algebra of $\mathbb{Z}_{n}$-graded TSs rely on the existence of a single (nonself-adjoint) symmetry generator $\mathcal{Q}$ and a grading operator $\tau$ satisfying

$[\tau, H]=0, \quad \tau^{n}=1$,

$\tau^{\dagger}=\tau^{-1}, \quad[\tau, \mathcal{Q}]_{q}=0$,

where $H$ is the Hamiltonian, $[,]_{q}$ is the $q$-bracket, and $q:=e^{2 \pi i / n}$. These equations follow from the requirement that the action of $\mathcal{Q}$ changes the color of a colored state by one unit.

Given the above definition of a TS and properties of the grading operator, we could show the following.

I. The algebra of the $\boldsymbol{Z}_{2}$-graded TS of type $(1,1)$ is precisely the algebra of $N=1$ supersymmetric quantum mechanics:

$$
[H, \mathcal{Q}]=0,
$$

$\left\{\mathcal{Q}, \mathcal{Q}^{\dagger}\right\}=2 H$

$\mathcal{Q}^{2}=0$.

II. The algebra of the $Z_{2}$-graded TS of type $(2,1)$ is precisely the algebra of parasupersymmetric quantum mechanics of Rubakov and Spiridonov:

$[H, \mathcal{Q}]=0$

$\left\{\mathcal{Q}^{2}, \mathcal{Q}^{\dagger}\right\}+\mathcal{Q} \mathcal{Q}^{\dagger} \mathcal{Q}=4 H \mathcal{Q}$

$\mathcal{Q}^{3}=0$

III. The algebra of the $\mathbb{Z}_{2}$-graded TS of type $\left(m_{+}, m_{-}\right)$, with $m_{-} \leq m_{+}$, is given by

$$
\begin{aligned}
& \left(\mathcal{Q}^{2}-\mathcal{K}_{1}\right)\left(\mathcal{Q}^{2}-\mathcal{K}_{2}\right) \cdots \\
& \left(\mathcal{Q}^{2}-\mathcal{K}_{m_{-}}\right) \mathcal{Q}^{1-\delta_{m_{-}, m_{+}}}=0 \\
& \left(Q_{j}^{2}-M_{j 1}\right)\left(Q_{j}^{2}-M_{j 2}\right) \cdots \\
& \left(Q_{j}^{2}-M_{j m_{-}}\right) Q_{j}^{1-\delta_{m_{-}, m_{+}}}=0,
\end{aligned}
$$

where $\mathcal{K}_{\ell}$ and $M_{j \ell}$ are central elements, $j \in$ $\{1,2\}$, and $Q_{j}$ are self-adjoint generators, i.e., $Q_{1}:=\left(\mathcal{Q}+\mathcal{Q}^{\dagger}\right) / \sqrt{2}$ and $Q_{2}:=(\mathcal{Q}-$ $\left.\mathcal{Q}^{\dagger}\right) /(i \sqrt{2})$.

IV. The algebra of the $\mathbb{Z}_{n}$-graded TS of type $(1,1, \cdots, 1)$ is given by $\mathcal{Q}^{n}=\mathcal{K}$, where $\mathcal{K}$ is a central element, and a couple of algebraic relations for $Q_{j}$ given in Ref. [5]. Note that identifying $\mathcal{K}$ with $H$, we obtain the algebra of fractional supersymmetry of order $n$.

V. The algebra of the $\mathbb{Z}_{n}$-graded TS of type $\left(m_{1}, m_{2}, \cdots, m_{n}\right)$, with $m_{1} \leq m_{2} \leq$ $\cdots \leq m_{n}$, is given by

$\left(\mathcal{Q}^{n}-\mathcal{K}_{1}\right)\left(\mathcal{Q}^{n}-\mathcal{K}_{2}\right) \cdots\left(\mathcal{Q}^{n}-\mathcal{K}_{m_{-}}\right) \mathcal{Q}^{1-\delta}=0$,

where $\mathcal{K}_{\ell}$ are central elements and $\delta$ is the multiplicity of $m_{1}$. In addition there are two algebraic relations for $Q_{j}$. 


\section{Conclusion}

We have obtained a generalization of supersymmetry that shares its topological properties. In our investigation of the algebraic structure of these symmetries we were led to a novel derivation of the algebras of supersymmetry, $(p=2)$ parasupersymmetry, and fractional supersymmetry.

We determined the algebra of the $\mathbb{Z}_{n}$-graded topological symmetries using the definition of a topological symmetry. Note however that in general the algebraic relations obtained in this way do not imply the desired degeneracy structures. In other words, topological symmetries belong to a proper subclass of symmetries obeying these relations.

For a specific model of $\mathbb{Z}_{n}$-graded TS of type $(1,1, \cdots, 1)$, we could relate the topological invariants $\Delta_{i j}$ to the differences of the dimensions of kernels of certain products of $n$ Fredholm operators. For details see Ref. [5].

More recently, we have explored the statistical origins of a class of TSs. In particular, we could construct concrete models possessing both $\mathbb{Z}_{2^{-}}$ graded TS of type $(1, n)$ and $\mathbb{Z}_{n}$-graded TS of type $(1,1, \cdots, 1)$ using bosons and orthofermions [6].

\section{REFERENCES}

1. V.A. Rubakov and V.P. Spiridonov, Parasupersymmetric Quantum Mechanics, Mod. Phys. Lett. A3 (1988) 1337.

2. A. Mostafazadeh, Spectrum Degeneracy of General $p=2$ Parasupersymmetric Quantum Mechanics and Parasupersymmetric Topological Invariants, Int. J. Mod. Phys. A11 (1996) 1057-1076, hep-th/9410180.

3. A. Mostafazadeh, Parasupersymmetric Quantum Mechanics and Indices of Fredholm Operators, Int. J. Mod. Phys. A12 (1997) 27252739 , hep-th/9603163.

4. A. Mostafazadeh and K. Aghababaei Samani, Topological Symmetries, Mod. Phys. Lett. A15 (2000) 175-184, hep-th/0003108.

5. K. Aghababaei Samani and A. Mostafazadeh, Quantum Mechanical Symmetries and Topo- logical Invariants, Nucl. Phys. B595 (2001) 467-492, hep-th/0007008.

6. K. Aghababaei Samani and A. Mostafazadeh, On the Statistical Origins of Topological Symmetries, hep-th/0105013. 